\title{
Coordinated control of spacecraft's attitude and end-effector for space robots
}

\author{
Alessandro M. Giordano ${ }^{1,2}$, Christian Ott $^{2}$, and Alin Albu-Schäffer ${ }^{1,2}$
}

\begin{abstract}
This paper addresses the coordinated control of the spacecraft's attitude and the end-effector pose of a manipulatorequipped space robot. A controller is proposed to simultaneously regulate the spacecraft's attitude, the global center-ofmass (CoM), and the end-effector pose. The control is based on a triangular actuation decomposition that decouples the endeffector task from the spacecraft's force actuator, increasing fuel efficiency. The strategy is validated in hardware using a robotic motion simulator composed of a seven degrees-of-freedom (DOF) arm mounted on a $6 \mathrm{DOF}$ base. The trade-off between control requirements and fuel consumption is discussed.
\end{abstract}

Index Terms-Space Robotics and Automation, Motion Control, Dynamics, Compliance and Impedance Control

\section{INTRODUCTION}

C ONCEPTUAL future orbital robotics systems envision a manipulator mounted on a spacecraft equipped with actuators. A typical configuration of spacecraft's actuators used in rendezvous scenarios includes at least thrusters, as they are the only devices that allow actuation of the translation. Thrusters are nonrenewable resources, as they rely upon the limited amount of fuel which has been launched with the spacecraft. Furthermore, their actuation capability is very small compared to the driving torques of the manipulator and they can be commanded at a consistently lower rate than the joints of the manipulator. The operational lifetime is strongly limited by the fuel limitation. Further, the manipulator performance is limited by the thrusters' saturation and, in coordinated control designs, by the discretization of the thrusters. Considering the above mentioned aspects, the derivation of intelligent control strategies that try to limit the use of the thrusters is a key point for the development of sustainable and high-performance orbital robotic systems.

In the early control concepts, attention was given to the possibility of completely turning off the spacecraft's actuators, resulting in a system for which the arm is commanded to realize an end-effector task while the spacecraft is left freefloating [1], [2], [3]. The free-floating idea was recently

Manuscript received: September, 10, 2018; Revised December, 5, 2018; Accepted January, 28, 2019.

This paper was recommended for publication by Editor Jonathan Roberts upon evaluation of the Associate Editor and Reviewers' comments.

${ }^{1}$ Alessandro M. Giordano and Alin Albu-Schäffer are with the Department of Informatics, Technische Universität München (TUM), Garching, 85748, Germany (email: alessandro.giordano@dlr.de; Alin.AlbuSchaeffer@dlr.de).

${ }^{2}$ Alessandro M. Giordano, Christian Ott, and Alin Albu-Schäffer are with the Institute of Robotics and Mechatronics, German Aerospace Center (DLR), 82234 Weßling, Germany (email: alessandro.giordano@dlr.de; christian.ott@dlr.de; Alin.Albu-Schaeffer@dlr.de).

Digital Object Identifier (DOI): see top of this page. extended in the sense that the spacecraft's actuators are not completely turned off, but they are (minimalistically) used to dump any accumulated linear and angular momenta from the system [4] and stabilize the center-of-mass (CoM) of the space robot [5], endowing the floating-base space robot with the capability to resist contact. Although the advantages of the free-floating control and its extensions are evident in terms of fuel consumption, some missions may still require attitude pointing of the spacecraft. Possible reasons for this might include the limited field-of-view of a spacecraftmounted sensor for relative navigation and antenna pointing for telecommunication. To cope with such constraints, the coordinated control of the spacecraft and of the arm endeffector was developed in the literature. For this purpose, the interesting strategy of fixed-attitude-restricted Jacobian control [6] was proposed. In the strategy, the simultaneous attitude and end-effector tasks are performed entirely by the manipulator joints and no thrusters are used. However, the method requires highly redundant robots and even in that case, the workspace of the robot might be too limited. Other strategies exploit the full actuation capability of a space robot [7], [8], [9] given by the combined use of the spacecraft's actuators and joint drives. In [7], an adaptive scheme is proposed for the control of the spacecraft's attitude and the joints; in [8] a feedback linearization scheme is proposed for the control of the spacecraft's attitude and the end-effector. However, in both works the stabilization of the inertial translational motion is not treated and the system may drift after contact. In [9], a coordinated control strategy is developed to simultaneously control the spacecraft translation, the spacecraft attitude, and the end-effector, based on a transposed Jacobian strategy. The method is effective in controlling both the spacecraft's attitude, the end-effector, and in stabilizing the inertial translational motion. However, the additional task of rigidly controlling the spacecraft's translation, as well as the coupled actuation structure resulting therein, lead to unnecessary activation of thrusters during end-effector maneuvering.

In this paper, a controller is designed to regulate the spacecraft's attitude and the end-effector pose while leaving the spacecraft free to translate. To stabilize the inertial motion of the robot, the space robot's CoM is controlled instead. One feature of the proposed controller is its decoupled actuation structure, i.e., the end-effector control input is decoupled from the spacecraft's force actuators. Thanks to this decoupling and to the avoidance of control of the spacecraft's translation, the controller improves the fuel efficiency compared to full spacecraft control, as demonstrated in a simulation comparison. The main contributions are: 
- the formulation of the dynamics in a transformed set of coordinates, for which the end-effector control input is decoupled from the spacecraft's force actuators;

- the development of a coordinated control of spacecraft attitude, global CoM, and end-effector pose, including a stability proof;

- an analysis of the trade-off between control requirements and fuel consumption for a space robot.

The paper is structured as follows: Section $\amalg$ introduces the notation and the main equations. Section [II describes the dynamics transformation and introduces the new decoupled control inputs. Section IV presents the proposed controller and the stability analysis. Section $\mathrm{V}$ discusses the trade-off between task requirements and fuel consumption. Section VI presents the experimental validation.

\section{Preliminaries}

\section{A. Problem statement}

A serial-link robot composed of $n+1$ bodies is considered, where $n$ is the number of joints of the arm. The spacecraft is fully actuated, i.e., external forces and torques are exerted on the spacecraft by means of the spacecraft actuators. The operational scenario involves the maneuvering of the robot's end-effector with the requirement of attitude control of the spacecraft but no requirement on the control control of its position. This may include the tasks of capturing, inspecting, or servicing a target object in orbit. The target object is assumed to be stationary in the inertial space. No orbital or environmental disturbances are considered, because they are considerably smaller than the actuation forces. The problem developed herein is the derivation of a controller that regulates simultaneously the robot's end-effector pose and the spacecraft's attitude but leaves the spacecraft free to translate. The control shall further regulate the position of the overall $\mathrm{CoM}$ to ensure no inertial drift and a favorable location of the workspace w.r.t. the target.

\section{B. Main notation}

Fig 1 shows a schematic representation of the system components. The following frames are considered: a base frame $\mathcal{B}$ attached to the spacecraft and placed on its $\mathrm{CoM}$; an endeffector frame $\mathcal{E}$; a frame $\mathcal{T}$ attached to the target object; a frame $\mathcal{C}$ placed on the $\mathrm{CoM}$ of the space robot and whose axes are nonrotating w.r.t. the inertial space; and a body frame $\mathcal{J}$ attached to the general $j^{\text {th }}$ body and placed on its CoM. Note that $j=0$ corresponds to the base frame. The symbols $\boldsymbol{f}_{b}$ and $\tau_{b}$ denote the base force and torque about $\mathcal{B}$ acted upon by the spacecraft's actuators and expressed in the frame $\mathcal{B}$. Let us indicate with $\boldsymbol{p}_{x y} \in \mathbb{R}^{3}$ and $\boldsymbol{R}_{x y} \in \mathrm{SO}(3)$ the position vector from a general frame $\mathcal{X}$ to a general frame $\mathcal{Y}$ expressed in the frame $\mathcal{X}$, and the corresponding rotation matrix, respectively. The so-called Adjoint transformation [10] is used herein:

$$
\boldsymbol{A}_{x y}=\left[\begin{array}{cc}
\boldsymbol{R}_{x y} & {\left[\boldsymbol{p}_{x y}\right]^{\wedge} \boldsymbol{R}_{x y}} \\
\mathbf{0} & \boldsymbol{R}_{x y}
\end{array}\right] \in \mathbb{R}^{6 \times 6},
$$

wherein the operator $[\cdot]^{\wedge}$ indicates the skew-symmetric matrix of the vector argument. Let us generally denote as $\boldsymbol{v}_{x y} \in$

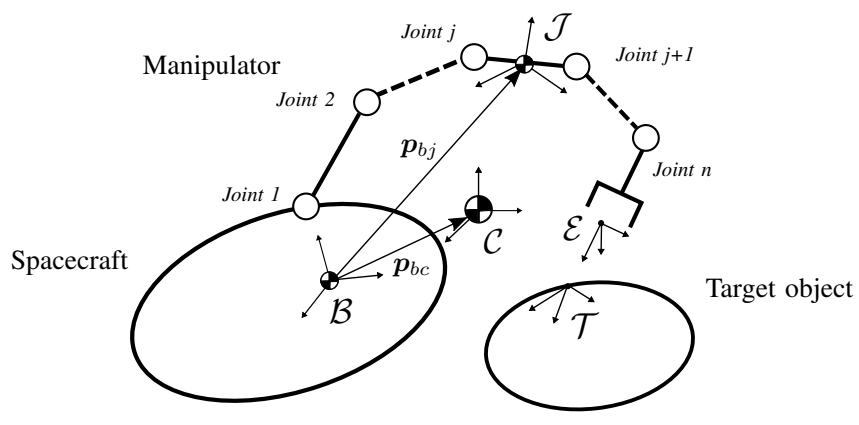

Fig. 1: Schematic representation of the system.

$\mathbb{R}^{3}$ and $\boldsymbol{\omega}_{x y} \in \mathbb{R}^{3}$ the linear and angular velocities of the general frame $\mathcal{Y}$ relative to $\mathcal{X}$ expressed in $\mathcal{Y}$, respectively. $\boldsymbol{\nu}_{x y}=\left[\begin{array}{ll}\boldsymbol{v}_{x y}^{T} & \boldsymbol{\omega}_{x y}^{T}\end{array}\right]^{T} \in \mathbb{R}^{6}$ indicates the corresponding 6DOF generalized velocity. The use of only one superscript, i.e., $\nu_{y}$, indicates that the velocity is relative to the inertial frame $\mathcal{T}$. The symbol $m^{(j)} \in \mathbb{R}$ denotes the mass of the $j^{t h}$ body, whereas $\boldsymbol{I}_{j}^{(j)} \in \mathbb{R}^{3}$ denotes its inertia around $\mathcal{J}$ expressed in the frame $\mathcal{J}$. The symbols $\mathbf{0}$ and $\boldsymbol{E}$ indicate the zero and identity matrices of suitable dimensions, respectively.

\section{Kinematics and dynamics}

The velocity of the $j^{t h}$ body can be expressed as a function of the base velocity and of the joint velocities, as

$$
\boldsymbol{\nu}_{j}=\boldsymbol{A}_{j b}(\boldsymbol{q})\left[\begin{array}{c}
\boldsymbol{v}_{b} \\
\boldsymbol{\omega}_{b}
\end{array}\right]+\boldsymbol{J}_{\nu_{j}}(\boldsymbol{q}) \dot{\boldsymbol{q}},
$$

where $\boldsymbol{q} \in \mathbb{T}^{n}$ and $\dot{\boldsymbol{q}} \in \mathbb{R}^{n}$ are the joint angle 1 and velocities, respectively, and where $\boldsymbol{J}_{\nu_{j}}(\boldsymbol{q})=\left[\begin{array}{l}\boldsymbol{J}_{v_{j}}(\boldsymbol{q}) \\ \boldsymbol{J}_{\omega_{j}}(\boldsymbol{q})\end{array}\right] \in \mathbb{R}^{6 \times n}$ is the Jacobian matrix mapping $\dot{\boldsymbol{q}}$ into $\boldsymbol{\nu}_{j}$, with $\boldsymbol{J}_{v_{j}}(\boldsymbol{q}) \in \mathbb{R}^{3 \times n}$ and $\boldsymbol{J}_{\omega_{j}}(\boldsymbol{q}) \in \mathbb{R}^{3 \times n}$ being its linear and angular parts, respectively. Note that for $j=0$, it holds that $\boldsymbol{A}_{j b}(\boldsymbol{q})=\boldsymbol{E}$ and $\boldsymbol{J}_{\nu_{j}}(\boldsymbol{q})=\mathbf{0}$. Similarly, the end-effector velocity is expressed as

$$
\boldsymbol{\nu}_{e}=\boldsymbol{A}_{e b}(\boldsymbol{q})\left[\begin{array}{l}
\boldsymbol{v}_{b} \\
\boldsymbol{\omega}_{b}
\end{array}\right]+\boldsymbol{J}_{\nu_{e}}(\boldsymbol{q}) \dot{\boldsymbol{q}}
$$

where $\boldsymbol{J}_{\nu_{e}}(\boldsymbol{q}) \in \mathbb{R}^{6 \times n}$ is the manipulator Jacobian matrix. The dynamics of the space robot is described by

$$
\begin{gathered}
\underbrace{\left[\begin{array}{ccc}
\boldsymbol{M}_{t} & \boldsymbol{M}_{t r} & \boldsymbol{M}_{t m} \\
\boldsymbol{M}_{t r}^{T} & \boldsymbol{M}_{r} & \boldsymbol{M}_{r m} \\
\boldsymbol{M}_{t m}^{T} & \boldsymbol{M}_{r m}^{T} & \boldsymbol{M}_{m}
\end{array}\right]}_{\boldsymbol{M}(\boldsymbol{q})}\left[\begin{array}{c}
\dot{\boldsymbol{v}}_{b} \\
\dot{\boldsymbol{\omega}}_{b} \\
\ddot{\boldsymbol{q}}
\end{array}\right]+ \\
+\underbrace{\left[\begin{array}{ccc}
\boldsymbol{C}_{t} & \boldsymbol{C}_{t r} & \boldsymbol{C}_{t m} \\
\boldsymbol{C}_{r t} & \boldsymbol{C}_{r} & \boldsymbol{C}_{r m} \\
\boldsymbol{C}_{m t} & \boldsymbol{C}_{m r} & \boldsymbol{C}_{m}
\end{array}\right]}_{\boldsymbol{C}\left(\boldsymbol{q}, \boldsymbol{v}_{b}, \boldsymbol{\omega}_{b}, \dot{\boldsymbol{q}}\right)}\left[\begin{array}{c}
\boldsymbol{v}_{b} \\
\boldsymbol{\omega}_{b} \\
\dot{\boldsymbol{q}}
\end{array}\right]=\left[\begin{array}{c}
\boldsymbol{f}_{b} \\
\boldsymbol{\tau}_{b} \\
\boldsymbol{\tau}
\end{array}\right],
\end{gathered}
$$

where $\boldsymbol{M}(\boldsymbol{q}) \in \mathbb{R}^{(6+n) \times(6+n)}$ and $\boldsymbol{C}\left(\boldsymbol{q}, \boldsymbol{v}_{b}, \boldsymbol{\omega}_{b}, \dot{\boldsymbol{q}}\right) \in$ $\mathbb{R}^{(6+n) \times(6+n)}$ are the inertia and Coriolis/centrifugal matrices, respectively, and where $\tau \in \mathbb{R}^{n}$ are the joint torques. Henceforth, the functional dependence is dropped out.

$$
{ }^{1} \mathbb{T}^{n}=\underbrace{\mathbb{S} \times \cdots \times \mathbb{S}}_{n} \text { is the } \mathrm{n} \text {-torus. }
$$


The sub-blocks of the inertia matrix are expressed as [5]

$$
\begin{aligned}
\boldsymbol{M}_{t} & =m \boldsymbol{E} \in \mathbb{R}^{3 \times 3}, \quad \boldsymbol{M}_{r}=\boldsymbol{I}_{b} \in \mathbb{R}^{3 \times 3}, \\
\boldsymbol{M}_{t r} & =-m\left[\boldsymbol{p}_{b c}\right]^{\wedge} \in \mathbb{R}^{3 \times 3}, \quad \boldsymbol{M}_{t m}=m \overline{\boldsymbol{J}}_{v} \in \mathbb{R}^{3 \times n}, \\
\boldsymbol{M}_{r m} & =\sum_{j=0}^{n} \boldsymbol{R}_{j b}^{T} \boldsymbol{I}_{j}^{(j)} \boldsymbol{J}_{\omega_{j}}+m^{(j)}\left[\boldsymbol{p}_{b j}\right]^{\wedge} \boldsymbol{R}_{j b}^{T} \boldsymbol{J}_{v_{j}} \in \mathbb{R}^{3 \times n}, \\
\boldsymbol{M}_{m} & =\sum_{j=0}^{n} m^{(j)} \boldsymbol{J}_{v_{j}}^{T} \boldsymbol{J}_{v_{j}}+\boldsymbol{J}_{\omega_{j}}^{T} \boldsymbol{I}_{j}^{(j)} \boldsymbol{J}_{\omega_{j}} \in \mathbb{R}^{n \times n},
\end{aligned}
$$

where $m=\sum_{j=0}^{n} m^{(j)}$ and $\boldsymbol{I}_{b}=\sum_{j=0}^{n} \boldsymbol{R}_{j b}^{T} \boldsymbol{I}_{j}^{(j)} \boldsymbol{R}_{j b}+$ $m^{(j)}\left[\boldsymbol{p}_{b j}\right]^{\wedge T}\left[\boldsymbol{p}_{b j}\right]^{\wedge}$ denote the mass of the whole system and its rotational inertia around $\mathcal{B}$, respectively, and where

$$
\begin{aligned}
\boldsymbol{p}_{b c} & =\frac{1}{m} \sum_{j=0}^{n} m_{j} \boldsymbol{p}_{b j} \in \mathbb{R}^{3}, \\
\overline{\boldsymbol{J}}_{v} & =\frac{1}{m} \sum_{j=0}^{n} m_{j} \boldsymbol{R}_{j b}^{T} \boldsymbol{J}_{v_{j}} \in \mathbb{R}^{3 \times n} .
\end{aligned}
$$

The velocity of the CoM of the whole system is computed as

$$
\boldsymbol{v}_{c}=\frac{1}{m} \sum_{j=0}^{n} m_{j} \boldsymbol{R}_{c j} \boldsymbol{v}_{j}
$$

To simplify (8), the linear part $\boldsymbol{v}_{j}$ is extracted from (2), as

$$
\boldsymbol{v}_{j}=\boldsymbol{R}_{j b} \boldsymbol{v}_{b}+\left[\boldsymbol{p}_{j b}\right]^{\wedge} \boldsymbol{R}_{j b} \boldsymbol{\omega}_{b}+\boldsymbol{J}_{v_{j}} \dot{\boldsymbol{q}},
$$

where (1) has been used. Then, by inserting (9) into (8) and using (6) and (7), $\boldsymbol{v}_{c}$ can be expressed as a function of the generalized velocities $\boldsymbol{v}_{b}, \boldsymbol{\omega}_{b}$ and $\dot{\boldsymbol{q}}$, as

$$
\boldsymbol{v}_{c}=\boldsymbol{R}_{c b} \boldsymbol{v}_{b}-\boldsymbol{R}_{c b}\left[\boldsymbol{p}_{b c}\right]^{\wedge} \boldsymbol{\omega}_{b}+\boldsymbol{R}_{c b} \overline{\boldsymbol{J}_{v}} \dot{\boldsymbol{q}} .
$$

\section{TRIANGULAR DYNAMICS}

In the following, the motion of the end-effector is first decomposed into a centroidal component plus a component of motion around the CoM. Then, a dynamics transformation is applied to identify a set of new control inputs that possess special decoupling properties with respect to the actuators.

\section{A. Circumcentroidal motion decomposition}

Let us first rewrite (3) more explicitly in translational and rotational base velocity and joint velocity components, as

$$
\boldsymbol{\nu}_{e}=\left[\begin{array}{c}
\boldsymbol{R}_{e b} \\
\mathbf{0}
\end{array}\right] \boldsymbol{v}_{b}+\left[\begin{array}{c}
{\left[\boldsymbol{p}_{e b}\right]^{\wedge} \boldsymbol{R}_{e b}} \\
\boldsymbol{R}_{e b}
\end{array}\right] \boldsymbol{\omega}_{b}+\boldsymbol{J}_{\nu_{e}} \dot{\boldsymbol{q}},
$$

where (1) was used. The end-effector velocity in (11) can be expressed as function of the CoM velocity by eliminating $\boldsymbol{v}_{b}$ from (10) and (11), obtaining 2

$$
\boldsymbol{\nu}_{e}=\boldsymbol{G}_{v_{c}} \boldsymbol{v}_{c}+\boldsymbol{G}_{\omega_{b}} \boldsymbol{\omega}_{b}+\boldsymbol{J}_{\nu_{e}}^{\oplus} \dot{\boldsymbol{q}},
$$

where:

$$
\begin{gathered}
\boldsymbol{G}_{v_{c}}=\left[\begin{array}{c}
\boldsymbol{R}_{e c} \\
\mathbf{0}
\end{array}\right] \in \mathbb{R}^{6 \times 3}, \quad \boldsymbol{G}_{\omega_{b}}=\left[\begin{array}{c}
{\left[\boldsymbol{p}_{e c}\right]^{\wedge} \boldsymbol{R}_{e b}} \\
\boldsymbol{R}_{e b}
\end{array}\right] \in \mathbb{R}^{6 \times 3}, \\
\boldsymbol{J}_{\nu_{e}}^{\oplus}=\boldsymbol{J}_{\nu_{e}}-\left[\begin{array}{c}
\boldsymbol{R}_{e b} \\
\mathbf{0}
\end{array}\right] \overline{\boldsymbol{J}}_{v} \in \mathbb{R}^{6 \times n} .
\end{gathered}
$$

\footnotetext{
${ }^{2}$ The property $\boldsymbol{R}[\boldsymbol{p}]^{\wedge} \boldsymbol{R}^{T}=[\boldsymbol{R} \boldsymbol{p}]^{\wedge}$ of the skew-symmetric matrices [10
} is used to obtain 12 .
Note that $\boldsymbol{J}_{\nu_{e}}^{\oplus}$ is the generalized manipulator Jacobian obtained by eliminating only the translational part of the base motion. Thus, it differs from the generalized Jacobian used for freefloating control [3], [5], which is obtained by eliminating both the translational and rotational parts. The end-effector velocity in (12) can be finally written as the sum of the motion of the $\mathrm{CoM}$ and that around the CoM, as

$$
\boldsymbol{\nu}_{e}=\boldsymbol{G}_{v_{c}} \boldsymbol{v}_{c}+\boldsymbol{\nu}_{e}^{\oplus}
$$

where $\boldsymbol{\nu}_{e}^{\oplus} \in \mathbb{R}^{6}$ refers to what in the following will be called "end-effector circumcentroidal motion," i.e., the motion around the overall CoM. It endows the effects of both the internal joint motion and the angular base motion, and it is given by

$$
\boldsymbol{\nu}_{e}^{\oplus} \triangleq \boldsymbol{G}_{\omega_{b}} \boldsymbol{\omega}_{b}+\boldsymbol{J}_{\nu_{e}}^{\oplus} \dot{\boldsymbol{q}}
$$

An interpretation of $\boldsymbol{\nu}_{e}^{\oplus}$ can be given in terms of relative motion of frames. More specifically, $\boldsymbol{\nu}_{e}^{\oplus}$ is equivalent to the body velocity $\boldsymbol{\nu}_{c e} \in \mathbb{R}^{6}$ of the end-effector frame relative to the nonrotating frame $\mathcal{C}$. In fact, given the velocity $\boldsymbol{\nu}_{c}=$ $\left[\begin{array}{ll}\boldsymbol{v}_{c}^{T} & \mathbf{0}^{T}\end{array}\right]^{T}$ of the frame $\mathcal{C}$, the body velocity $\boldsymbol{\nu}_{c e} \in \mathbb{R}^{6}$ of $\mathcal{E}$ relative to $\mathcal{C}$ is [10, p.59]

$$
\boldsymbol{\nu}_{c e}=\boldsymbol{\nu}_{e}-\boldsymbol{A}_{e c} \boldsymbol{\nu}_{c}=\boldsymbol{\nu}_{e}-\left[\begin{array}{c}
\boldsymbol{R}_{e c} \\
\mathbf{0}
\end{array}\right] \boldsymbol{v}_{c},
$$

where (1) was used. Rearranging (15) and using (13), it is

$$
\boldsymbol{\nu}_{e}^{\oplus}=\boldsymbol{\nu}_{e}-\boldsymbol{G}_{v_{c}} \boldsymbol{v}_{c}=\boldsymbol{\nu}_{e}-\left[\begin{array}{c}
\boldsymbol{R}_{e c} \\
\mathbf{0}
\end{array}\right] \boldsymbol{v}_{c},
$$

which is indeed equivalent to (17). In the following section it is shown how $\boldsymbol{\nu}_{e}^{\oplus}$ possesses special properties that are useful to decouple the dynamics equations.

\section{B. Dynamics transformation}

A coordinates transformation $\boldsymbol{\Gamma} \in \mathbb{R}^{12 \times(6+n)}$ can be defined based on the circumcentroidal motion decomposition, as

$$
\left[\begin{array}{c}
\boldsymbol{v}_{c} \\
\boldsymbol{\omega}_{b} \\
\boldsymbol{\nu}_{e}^{\oplus}
\end{array}\right]=\underbrace{\left[\begin{array}{ccc}
\boldsymbol{R}_{c b} & -\boldsymbol{R}_{c b}\left[\boldsymbol{p}_{b c}\right]^{\wedge} & \boldsymbol{R}_{c b} \overline{\boldsymbol{J}}_{v} \\
\mathbf{0} & \boldsymbol{E} & \mathbf{0} \\
\mathbf{0} & \boldsymbol{G}_{\omega_{b}} & \boldsymbol{J}_{\nu_{e}}^{\oplus}
\end{array}\right]}_{\boldsymbol{\Gamma}}\left[\begin{array}{c}
\boldsymbol{v}_{b} \\
\boldsymbol{\omega}_{b} \\
\dot{\boldsymbol{q}}
\end{array}\right],
$$

where (10) and (16) have been used. The generalized forces transform as

$$
\left[\begin{array}{c}
\boldsymbol{f}_{b} \\
\boldsymbol{\tau}_{b} \\
\boldsymbol{\tau}
\end{array}\right]=\boldsymbol{\Gamma}^{T}\left[\begin{array}{c}
\boldsymbol{f}_{c} \\
\boldsymbol{\tau}_{b}^{\oplus} \\
\boldsymbol{w}_{e}^{\oplus}
\end{array}\right],
$$

where $\boldsymbol{f}_{c} \in \mathbb{R}^{3}, \boldsymbol{\tau}_{b}^{\oplus} \in \mathbb{R}^{3}$ and $\boldsymbol{w}_{e}^{\oplus} \in \mathbb{R}^{6}$ are the new control inputs. $f_{c}$ represents the total CoM control force, $\tau_{b}^{\oplus}$ is the new base control torque and $\boldsymbol{w}_{e}^{\oplus}$ is the new end-effector control wrench. Let us assume a nonredundant manipulator, i.e., $n=6$. For nonsingular $\boldsymbol{J}_{\nu_{e}}^{\oplus}$ it is possible to invert (19) and transform (4) as [11, p.32]:

$$
\begin{gathered}
{\left[\begin{array}{ccc}
m \boldsymbol{E} & \mathbf{0} & \mathbf{0} \\
\mathbf{0} & \breve{\boldsymbol{M}}_{b} & \breve{\boldsymbol{M}}_{b e} \\
\mathbf{0} & \breve{\boldsymbol{M}}_{b e}^{T} & \breve{\boldsymbol{M}}_{e}
\end{array}\right]\left[\begin{array}{c}
\dot{\boldsymbol{v}}_{c} \\
\dot{\boldsymbol{\omega}}_{b} \\
\dot{\boldsymbol{\nu}}_{e}^{\oplus}
\end{array}\right]+} \\
+\left[\begin{array}{ccc}
\mathbf{0} & -\boldsymbol{C}_{b c}^{T} & -\boldsymbol{C}_{e c}^{T} \\
\boldsymbol{C}_{b c} & \breve{\boldsymbol{C}}_{b} & \breve{\boldsymbol{C}}_{b e} \\
\boldsymbol{C}_{e c} & \breve{\boldsymbol{C}}_{e b} & \breve{\boldsymbol{C}}_{e}
\end{array}\right]\left[\begin{array}{c}
\boldsymbol{v}_{c} \\
\boldsymbol{\omega}_{b} \\
\boldsymbol{\nu}_{e}^{\oplus}
\end{array}\right]=\left[\begin{array}{c}
\boldsymbol{f}_{c} \\
\boldsymbol{\tau}_{b}^{\oplus} \\
\boldsymbol{w}_{e}^{\oplus}
\end{array}\right],
\end{gathered}
$$


where $\breve{\boldsymbol{M}}=\left[\begin{array}{cc}\breve{\boldsymbol{M}}_{b} & \breve{\boldsymbol{M}}_{b e} \\ \breve{\boldsymbol{M}}_{b e}^{T} & \breve{\boldsymbol{M}}_{e}\end{array}\right] \in \mathbb{R}^{9 \times 9}$ and $\breve{\boldsymbol{C}}=\left[\begin{array}{cc}\breve{\boldsymbol{C}}_{b} & \breve{\boldsymbol{C}}_{b e} \\ \breve{\boldsymbol{C}}_{e b} & \breve{\boldsymbol{C}}_{e}\end{array}\right] \in$ $\mathbb{R}^{9 \times 9}$ are the inertia and Coriolis-centrifugal matrices, respectively, associated to the attitude and end-effector system. Notice that the CoM equation is inertially decoupled from the rest of the system. Note that this energy decoupling does not hold when using $\boldsymbol{\nu}_{e}$ instead of $\boldsymbol{\nu}_{e}^{\oplus}$. Further simplifications of (21) are done by considering that the Coriolis and centrifugal vector terms can be shown to be zero for the centroid equation, i.e., $-C_{b c}^{T} \boldsymbol{\omega}_{b}-\boldsymbol{C}_{e c}^{T} \boldsymbol{\nu}_{e}^{\oplus}=\mathbf{0}$ (see Appendix of [4]). Then, (21) simplifies to

$$
\begin{aligned}
& m \dot{\boldsymbol{v}}_{c}=\boldsymbol{f}_{c}, \\
& \breve{\boldsymbol{M}}\left[\begin{array}{c}
\dot{\boldsymbol{\omega}}_{b} \\
\dot{\boldsymbol{\nu}}_{e}^{\oplus}
\end{array}\right]+\breve{\boldsymbol{C}}\left[\begin{array}{c}
\boldsymbol{\omega}_{b} \\
\boldsymbol{\nu}_{e}^{\oplus}
\end{array}\right]+\boldsymbol{C}_{c} \boldsymbol{v}_{c}=\left[\begin{array}{c}
\boldsymbol{\tau}_{b}^{\oplus} \\
\boldsymbol{w}_{e}^{\oplus}
\end{array}\right],
\end{aligned}
$$

where $\boldsymbol{C}_{c}=\left[\begin{array}{l}\boldsymbol{C}_{b c} \\ \boldsymbol{C}_{e c}\end{array}\right] \in \mathbb{R}^{6 \times 3}$. First, notice that the left-hand sides of (22a) and (22b) have a triangular structure, wherein $\boldsymbol{C}_{c} \boldsymbol{v}_{c}$ represents the perturbation of the CoM system into the coupled base and end-effector system. By exploiting this triangular structure it is possible to design controllers in a cascaded fashion, using $\left[\begin{array}{c}\tau_{b}^{\oplus} \\ \boldsymbol{w}_{e}^{\oplus}\end{array}\right]$ to control the base and the endeffector in a coordinated way, and using $f_{c}$ to independently control the CoM. Thanks to this triangular structure, the proof of stability can be addressed in cascade, as shown in the next section.

Second, remark that (22b) enjoys the fruitful property

$$
\left[\begin{array}{ll}
\boldsymbol{\omega}_{b}^{T} & \boldsymbol{\nu}_{e}^{\oplus T}
\end{array}\right](\dot{\dot{M}}-2 \breve{C})\left[\begin{array}{c}
\boldsymbol{\omega}_{b} \\
\boldsymbol{\nu}_{e}^{\oplus}
\end{array}\right]=\mathbf{0}, \forall \boldsymbol{\omega}_{b}, \boldsymbol{\nu}_{e}^{\oplus} \in \mathbb{R}^{6} .
$$

This property is an advantage of the machinery (19), (20), (21) used to obtain (22). Indeed, while 22a) is a common result, the advantage lies instead in (22b), for which (23) automatically holds.

\section{CoORdinated CONTROL}

The objective of the control is to regulate the pose of the frame $\mathcal{E}$, the position of $\mathcal{C}$ and the orientation of the frame $\mathcal{B}$ w.r.t. the inertial frame $\mathcal{T}$.

\section{A. Control error definition}

Given a desired CoM position $\boldsymbol{p}_{t c_{d}} \in \mathbb{R}^{3}$ fixed in $\mathcal{T}$, a CoM error $\tilde{\boldsymbol{x}}_{c} \in \mathbb{R}^{3}$ is defined as $\tilde{\boldsymbol{x}}_{c}=\boldsymbol{p}_{c c_{d}}=\boldsymbol{R}_{t c}^{T}\left(\boldsymbol{p}_{t c}-\boldsymbol{p}_{t c_{d}}\right)$ and its time-derivative is simply $\dot{\tilde{\boldsymbol{x}}}_{c}=\boldsymbol{R}_{t c}^{T} \dot{\boldsymbol{p}}_{t c}=\boldsymbol{v}_{c}$. Given a desired frame $\mathcal{E}_{d}$ fixed in $\mathcal{T}$, let us consider the position vector $\boldsymbol{p}_{t e_{d}}$ and the rotation matrix $\boldsymbol{R}_{t e_{d}}$ from $\mathcal{T}$ to $\mathcal{E}_{d}$. Then, an end-effector pose error $\tilde{\boldsymbol{x}}_{e} \in \mathbb{R}^{6}$ is defined using a quaternion-based coordinates representation, as $\tilde{\boldsymbol{x}}_{e}=\left[\begin{array}{ll}\boldsymbol{p}_{e e_{d}}^{T} & 2 \boldsymbol{\epsilon}_{e e_{d}}^{T}\end{array}\right]^{T}$, where $\boldsymbol{p}_{e e_{d}}=\boldsymbol{R}_{t e}^{T}\left(\boldsymbol{p}_{t e}-\boldsymbol{p}_{t e_{d}}\right)$ and where $\boldsymbol{\epsilon}_{e e_{d}} \in \mathbb{R}^{3}$ is the vector part of the unit quaternion extracted from $\boldsymbol{R}_{e e_{d}}=\boldsymbol{R}_{t e}^{T} \boldsymbol{R}_{t e_{d}}$. Denoting by $\eta_{e e_{d}} \in \mathbb{R}$ its scalar part, the time derivative $\dot{\tilde{\boldsymbol{x}}}_{e}$ can be expressed as

$$
\dot{\tilde{\boldsymbol{x}}}_{e}=\boldsymbol{J}_{\tilde{x}_{e}} \boldsymbol{\nu}_{e}, \quad \text { with } \boldsymbol{J}_{\tilde{x}_{e}}=\left[\begin{array}{cc}
\boldsymbol{E} & \mathbf{0} \\
\mathbf{0} & -\eta_{e e_{d}} \boldsymbol{E}+\left[\boldsymbol{\epsilon}_{e e_{d}}\right]^{\wedge}
\end{array}\right] \text {, }
$$

where $\boldsymbol{J}_{\tilde{x}_{e}} \in \mathbb{R}^{6 \times 6}$ is the so-called coordinates representation Jacobian matrix. An advantage of the adopted representation is that $\boldsymbol{J}_{\tilde{x}_{e}}$ is not affected by singularity, i.e., $\boldsymbol{J}_{\tilde{x}_{e}}$ cannot grow unbounded, and that it is task-consistent [12, p.13]. Similarly, given a desired frame $\mathcal{B}_{d}$ with axes fixed in $\mathcal{T}$, let us consider the rotation matrix $\boldsymbol{R}_{t b_{d}}$ from $\mathcal{T}$ to $\mathcal{B}_{d}$. Then, a base attitude error $\tilde{\boldsymbol{x}}_{b} \in \mathbb{R}^{3}$ is defined as $\tilde{\boldsymbol{x}}_{b}=2 \boldsymbol{\epsilon}_{b b_{d}}$, with $\boldsymbol{\epsilon}_{b b_{d}} \in \mathbb{R}^{3}$ being the vector part of the unit quaternion extracted from $\boldsymbol{R}_{b b_{d}}=\boldsymbol{R}_{t b}^{T} \boldsymbol{R}_{t b_{d}}$. Denoting by $\eta_{b b_{d}} \in \mathbb{R}$ the scalar part of the quaternion, the time derivative $\dot{\tilde{\boldsymbol{x}}}_{b}$ can be expressed as

$\dot{\tilde{\boldsymbol{x}}}_{b}=\boldsymbol{J}_{\tilde{x}_{b}} \boldsymbol{\omega}_{b}, \quad$ with $\quad \boldsymbol{J}_{\tilde{x}_{b}}=-\eta_{b b_{d}} \boldsymbol{E}+\left[\boldsymbol{\epsilon}_{b b_{d}}\right]^{\wedge} \in \mathbb{R}^{3 \times 3}$.

For convenience, the base and end-effector errors (25) and (24) are rewritten in a compact form, as

$$
\dot{\tilde{\boldsymbol{x}}}=\boldsymbol{J}_{\tilde{x}}\left[\begin{array}{l}
\boldsymbol{\omega}_{b} \\
\boldsymbol{\nu}_{e}
\end{array}\right],
$$

where $\tilde{\boldsymbol{x}}=\left[\begin{array}{c}\tilde{\boldsymbol{x}}_{b} \\ \tilde{\boldsymbol{x}}_{e}\end{array}\right] \in \mathbb{R}^{9}$ and $\boldsymbol{J}_{\tilde{x}}=\left[\begin{array}{cc}\boldsymbol{J}_{\tilde{x}_{b}} & \mathbf{0} \\ \mathbf{0} & \boldsymbol{J}_{\tilde{x}_{e}}\end{array}\right] \in \mathbb{R}^{9 \times 9}$. Then, by inserting (15) into (26), $\dot{\tilde{x}}$ can be factored as

$$
\dot{\tilde{\boldsymbol{x}}}=\boldsymbol{J}_{\tilde{x}} \breve{\boldsymbol{v}}+\boldsymbol{J}_{\tilde{x}} \breve{\boldsymbol{G}}_{v_{c}} \boldsymbol{v}_{c}
$$

where $\breve{\boldsymbol{v}}=\left[\begin{array}{c}\boldsymbol{\omega}_{b} \\ \boldsymbol{\nu}_{e}^{\oplus}\end{array}\right] \in \mathbb{R}^{9}$ and $\breve{\boldsymbol{G}}_{v_{c}}=\left[\begin{array}{c}\mathbf{0} \\ \boldsymbol{G}_{v_{c}}\end{array}\right] \in \mathbb{R}^{9 \times 3}$.

\section{B. Controller design}

The CoM controller is defined as

$$
\boldsymbol{f}_{c}=-\boldsymbol{K}_{c} \tilde{\boldsymbol{x}}_{c}-\boldsymbol{D}_{c} \boldsymbol{v}_{c}
$$

where $\boldsymbol{K}_{c} \in \mathbb{R}^{3 \times 3}$ is a symmetric, positive definite stiffness matrix, and $\boldsymbol{D}_{c} \in \mathbb{R}^{3 \times 3}$ is a positive definite damping matrix. Then, the base controller is defined as

$$
\boldsymbol{\tau}_{b}^{\oplus}=-\boldsymbol{J}_{\tilde{x}_{b}}^{T} \boldsymbol{K}_{b} \tilde{\boldsymbol{x}}_{b}-\boldsymbol{D}_{b} \boldsymbol{\omega}_{b},
$$

where $\boldsymbol{K}_{b} \in \mathbb{R}^{3 \times 3}$ is a symmetric, positive definite stiffness matrix, and $\boldsymbol{D}_{b} \in \mathbb{R}^{3 \times 3}$ is a positive definite damping matrix. The end-effector controller is defined as

$$
\boldsymbol{w}_{e}^{\oplus}=-\boldsymbol{J}_{\tilde{x}_{e}}^{T} \boldsymbol{K}_{e} \tilde{\boldsymbol{x}}_{e}-\boldsymbol{D}_{e} \boldsymbol{\nu}_{e},
$$

where $\boldsymbol{K}_{e} \in \mathbb{R}^{6 \times 6}$ is a symmetric, positive definite stiffness matrix, and $\boldsymbol{D}_{e} \in \mathbb{R}^{6 \times 6}$ is a positive definite damping matrix. The controllers (28), (29), and (30) can be all interpreted as springs and dampers in the inertial space actuated by control inputs $\boldsymbol{f}_{c}, \boldsymbol{\tau}_{b}^{\oplus}$ and $\boldsymbol{w}_{e}^{\oplus}$ that are dual to the new space (19). For the sake of compactness, the base and end-effector controllers (29) and (30) are rewritten in the form

$$
\left[\begin{array}{c}
\boldsymbol{\tau}_{b}^{\oplus} \\
\boldsymbol{w}_{e}^{\oplus}
\end{array}\right]=\boldsymbol{J}_{\tilde{x}}^{T} \breve{\boldsymbol{K}} \tilde{\boldsymbol{x}}+\breve{\boldsymbol{D}} \breve{\boldsymbol{v}}
$$

where $\breve{\boldsymbol{K}}=\operatorname{blkdiag}\left(\boldsymbol{K}_{b}, \boldsymbol{K}_{e}\right) \in \mathbb{R}^{9 \times 9}$ and $\breve{\boldsymbol{D}}=$ $\operatorname{blkdiag}\left(\boldsymbol{D}_{b}, \boldsymbol{D}_{e}\right) \in \mathbb{R}^{9 \times 9}$. 


\section{Controller properties}

Writing (20) explicitly, the actuator commands can be related to the new control inputs as:

$$
\left[\begin{array}{c}
\boldsymbol{f}_{b} \\
\boldsymbol{\tau}_{b} \\
\boldsymbol{\tau}
\end{array}\right]=\left[\begin{array}{ccc}
\boldsymbol{R}_{c b}^{T} & \mathbf{0} & \mathbf{0} \\
{\left[\boldsymbol{p}_{b c}\right]^{\wedge} \boldsymbol{R}_{c b}^{T}} & \boldsymbol{E} & \boldsymbol{G}_{\omega_{b}}^{T} \\
\overline{\boldsymbol{J}}_{v}^{T} \boldsymbol{R}_{c b}^{T} & \mathbf{0} & \boldsymbol{J}_{\nu_{e}}^{\oplus T}
\end{array}\right]\left[\begin{array}{c}
\boldsymbol{f}_{c} \\
\boldsymbol{\tau}_{b}^{\oplus} \\
\boldsymbol{w}_{e}^{\oplus}
\end{array}\right] .
$$

Notice that the actuation distribution has a triangular form. More specifically, the base force $f_{b}$ is only activated to control the CoM position and not to actuate either the end-effector or the base attitude task. Conversely, the base torque $\tau_{b}$ and the joint torques $\tau$ are affected by $f_{c}$. One important feature of this triangular structure is that the base force actuator is not used to realize the end-effector task, but to realize the only task that cannot be actuated by internal actuators, i.e., controlling the inertial location of the CoM. This structure is a property of the circumcentroidal velocity $\boldsymbol{\nu}_{e}^{\oplus}$ and does not hold when using the absolute velocity $\boldsymbol{\nu}_{e}$. In fact, in the latter case, it would be:

$$
\left[\begin{array}{c}
\boldsymbol{f}_{b} \\
\boldsymbol{\tau}_{b} \\
\boldsymbol{\tau}
\end{array}\right]=\left[\begin{array}{ccc}
\boldsymbol{R}_{c b}^{T} & \mathbf{0} & \boldsymbol{P}_{v_{b}}^{T} \\
{\left[\boldsymbol{p}_{b c} c^{\wedge} \boldsymbol{R}_{c b}^{T}\right.} & \boldsymbol{E} & \boldsymbol{P}_{\omega_{b}}^{T} \\
\overline{\boldsymbol{J}}_{v}^{T} \boldsymbol{R}_{c b}^{T} & \mathbf{0} & \boldsymbol{J}_{\nu_{e}}^{T}
\end{array}\right]\left[\begin{array}{c}
\boldsymbol{f}_{c} \\
\overline{\boldsymbol{\tau}}_{b} \\
\boldsymbol{w}_{e}
\end{array}\right],
$$

where $\boldsymbol{P}_{v_{b}}, \boldsymbol{P}_{\omega_{b}} \in \mathbb{R}^{6 \times 3}$, and where $\overline{\boldsymbol{\tau}}_{b}, \boldsymbol{w}_{e} \in \mathbb{R}^{n}$ are control inputs dual to $\boldsymbol{\omega}_{b}, \boldsymbol{\nu}_{e}$. Another important feature can be concluded based on the special conserving properties of the CoM task. After the CoM transient vanishes, the system converges to a stationary situation in which the CoM remains fixed in the inertial space. Therefore, during the entire time of robot maneuvers that do not involve contact or $\mathrm{CoM}$ relocation, it will remain $\tilde{\boldsymbol{x}}_{c}=\boldsymbol{v}_{c}=\mathbf{0}$ and in turn $\boldsymbol{f}_{b}=\mathbf{0}$. Hence, with the proposed controller, all operations that do not involve contact will require no base force. The base force will be activated only when contact occurs, and its use will be limited to restoring the CoM location for the workspace need. In designs in which thrusters are used to actuate both $f_{b}$ and $\tau_{b}$, the above-mentioned features result in a consistent improvement in fuel consumption, as will be shown in Section V] In designs in which the thrusters are used to actuate only $f_{b}$, but the actuation of $\tau_{b}$ is accomplished by momentum exchange devices (e.g., reaction wheels), the proposed control would have the remarkable advantage of consuming exactly zero fuel for contact-free end-effector maneuvering.

\section{Stability analysis}

The closed-loop dynamics is obtained by inserting (31), (28) and (15) into (22), considering (27), and inverting (16), as:

$$
\begin{aligned}
& m \ddot{\tilde{\boldsymbol{x}}}_{c}+\boldsymbol{D}_{c} \dot{\tilde{\boldsymbol{x}}}_{c}+\boldsymbol{K}_{c} \tilde{\boldsymbol{x}}_{c}=\mathbf{0}, \\
& \breve{\boldsymbol{M}}(\boldsymbol{q}) \dot{\boldsymbol{v}}+\breve{\boldsymbol{C}}\left(\boldsymbol{q}, \breve{\boldsymbol{v}}, \dot{\tilde{\boldsymbol{x}}}_{c}\right) \breve{\boldsymbol{v}}+\breve{\boldsymbol{D}} \breve{\boldsymbol{v}}+\boldsymbol{J}_{\tilde{x}}^{T}(\tilde{\boldsymbol{x}}) \breve{\boldsymbol{K}} \tilde{\boldsymbol{x}} \\
& =-\left(\boldsymbol{C}_{c}\left(\boldsymbol{q}, \breve{\boldsymbol{v}}, \dot{\tilde{\boldsymbol{x}}}_{c}\right)+\breve{\boldsymbol{D}} \breve{G}_{v_{c}}(\boldsymbol{q})\right) \dot{\tilde{\boldsymbol{x}}}_{c}, \\
& \dot{\tilde{\boldsymbol{x}}}=\boldsymbol{J}_{\tilde{x}}(\tilde{\boldsymbol{x}}) \breve{\boldsymbol{v}}+\boldsymbol{J}_{\tilde{x}}(\tilde{\boldsymbol{x}}) \breve{\boldsymbol{G}}_{v_{c}}(\boldsymbol{q}) \dot{\tilde{\boldsymbol{x}}}_{c} . \\
& \dot{\boldsymbol{q}}=\boldsymbol{J}_{\nu_{e}}^{\oplus-1}(\boldsymbol{q}) \breve{G}_{\omega_{b}}(\boldsymbol{q}) \breve{\boldsymbol{v}},
\end{aligned}
$$

where $\breve{\boldsymbol{G}}_{\omega_{b}}=\left[\begin{array}{ll}\boldsymbol{E} & -\boldsymbol{G}_{\omega_{b}}\end{array}\right] \in \mathbb{R}^{6 \times 9}$. The state can be partitioned as $\boldsymbol{z}=\left[\begin{array}{ll}\boldsymbol{z}_{1}^{T} & \boldsymbol{z}_{2}^{T}\end{array}\right]^{T} \in D=\mathbb{R}^{24} \times \mathbb{T}^{n}$, with $\boldsymbol{z}_{1}=$
$\left[\begin{array}{ll}\tilde{\boldsymbol{x}}_{c}^{T} & \dot{\tilde{\boldsymbol{x}}}_{c}^{T}\end{array}\right]^{T} \in \mathbb{R}^{6}$ and $\boldsymbol{z}_{2}=\left[\begin{array}{lll}\breve{\boldsymbol{v}}^{T} & \tilde{\boldsymbol{x}}^{T} & \boldsymbol{q}^{T}\end{array}\right]^{T} \in \mathbb{R}^{18} \times \mathbb{T}^{n}$. Then, the dynamics (34) is in the cascade state-space form

$$
\begin{aligned}
& \dot{\boldsymbol{z}}_{1}=\boldsymbol{g}_{1}\left(\boldsymbol{z}_{1}\right), \\
& \dot{\boldsymbol{z}}_{2}=\boldsymbol{g}_{2}\left(\boldsymbol{z}_{1}, \boldsymbol{z}_{2}\right),
\end{aligned}
$$

where $g_{1}$ is obtained from (34a) and $g_{2}$ from (34b), 34c and $(34 \mathrm{~d})$. Notice that the dynamics of $\boldsymbol{z}_{1}$ is totally decoupled from the rest of the state and, furthermore, is linear. Let us define a region $\Omega$ that excludes the singularities of $\boldsymbol{J}_{\nu_{e}}^{\oplus}(\boldsymbol{q})$, as

$$
\Omega=\left\{\boldsymbol{z} \in D: \sigma_{\min }\left(\boldsymbol{J}_{\nu_{e}}^{\oplus}(\boldsymbol{q})\right)>0\right\},
$$

where $\sigma_{\min }(\cdot)$ indicates the minimum singular value of a matrix. In the region $\Omega$, the dynamics matrices $\breve{M}, \breve{C}$, and $\boldsymbol{C}_{c}$ exist.

Proposition IV.1. The invariant set $\overline{\boldsymbol{z}}=\left\{\boldsymbol{z} \in \Omega: \tilde{\boldsymbol{x}}_{c}=\dot{\tilde{\boldsymbol{x}}}_{c}=\right.$ $\mathbf{0}, \tilde{\boldsymbol{x}}=\breve{\boldsymbol{v}}=\mathbf{0}\}$ is asymptotically stable.

Proof. $\bar{z}$ is compact because $\mathbb{T}^{n}$ is compact. Then, cascade theorems for compact invariant sets [13] apply. The proof is done in cascade, proving first the stability of (35a) and then that of $35 \mathrm{~b}$ ) with $\boldsymbol{z}_{1}=\mathbf{0}$.

1) The system (34a) is asymptotically stable, having chosen $\boldsymbol{K}_{c}$ and $\boldsymbol{D}_{c}$ as positive definite. Therefore the subsystem $\dot{\boldsymbol{z}}_{1}=\boldsymbol{g}_{1}\left(\boldsymbol{z}_{1}\right)$ is asymptotically stable.

2) The stability of the subsystem $\dot{z}_{2}=\boldsymbol{g}_{2}\left(\mathbf{0}, \boldsymbol{z}_{2}\right)$ is addressed using the Lyapunov function

$$
V=\frac{1}{2} \breve{\boldsymbol{v}}^{T} \breve{\boldsymbol{M}} \breve{\boldsymbol{v}}+\frac{1}{2} \tilde{\boldsymbol{x}}^{T} \breve{\boldsymbol{K}} \tilde{\boldsymbol{x}}>0, \quad \forall \boldsymbol{z} \notin \overline{\boldsymbol{z}},
$$

which is always defined in $\Omega$. The time derivative along the system trajectories is

$$
\begin{aligned}
\dot{V}= & \breve{\boldsymbol{v}}^{T} \breve{\boldsymbol{M}} \dot{\boldsymbol{v}}+\frac{1}{2} \breve{\boldsymbol{v}}^{T} \dot{\boldsymbol{M}} \breve{\boldsymbol{v}}+\breve{\boldsymbol{v}}^{T} \boldsymbol{J}_{\tilde{x}}^{T} \breve{\boldsymbol{K}} \tilde{\boldsymbol{x}}= \\
& \frac{1}{2} \breve{\boldsymbol{v}}^{T}(\dot{\dot{\boldsymbol{M}}}-2 \breve{\boldsymbol{C}}) \breve{\boldsymbol{v}}-\breve{\boldsymbol{v}}^{T} \breve{\boldsymbol{D}} \breve{\boldsymbol{v}}=-\breve{\boldsymbol{v}}^{T} \breve{\boldsymbol{D}} \breve{\boldsymbol{v}} \leq 0,
\end{aligned}
$$

where (34b) and (34c) were used with $\dot{\tilde{x}}_{c}=\mathbf{0}$ and where the property (23) was exploited. Applying LaSalle to (34b), $\breve{\boldsymbol{v}} \equiv \mathbf{0}$ implies $\tilde{\boldsymbol{x}}=\mathbf{0}$ and the asymptotic stability of $\dot{\boldsymbol{z}}_{2}=\boldsymbol{g}_{2}\left(\mathbf{0}, \boldsymbol{z}_{2}\right)$ is thus proven.

From 1) and 2) then follows the asymptotic stability of the closed-loop (35).

\section{TRADE-OFF BETWEEN REQUIREMENTS AND FUEL CONSUMPTION}

To outline the advantage of the proposed control, a comparison is performed with two different strategies that enforce different requirements on the base motion. A simulative comparison is performed under ideal conditions for the following control methods:

1) Full base control [9]: the base translation and rotation are controlled using base actuators.

2) Partial base control (proposed): the base attitude is controlled and the base translation is left free. The space robot's CoM is controlled instead.

3) Floating-base control [5]: both the base translation and rotation are left free. The space robot's CoM and the angular momentum are controlled instead. 
A representative end-effector maneuver in a capture scenario is tested. The end-effector is commanded to reach a desired target pose, while the base is commanded according to the different requirements of the three different control methods. The end-effector command is generated using a smooth trajectory, while the base (and system's CoM) commands are constant setpoints. No contact or initial momentum are simulated. The simulated arm is a KUKA KR4+ lightweight robot which weights approximately $17 \mathrm{~kg}$. The dynamics parameters for the base are: mass $m^{(0)}=150 \mathrm{~kg}$, inertia $I_{0}^{(0)}=$ blkdiag $(21.8,15,18.88) \mathrm{kgm}^{2}$. The three controllers are compared in terms of functional behavior and fuel consumption. The gains used are the same for all $\mathrm{x}, \mathrm{y}$, and $\mathrm{z}$ components and are $k_{e, \text { trasl }}=800 \mathrm{~N} \mathrm{~m}^{-1}, k_{e, \text { rot }}=56 \mathrm{Nm} \mathrm{rad}^{-1}, k_{b, \text { trasl }}=$ $1000 \mathrm{~N} \mathrm{~m}^{-1}, k_{b, r o t}=672 \mathrm{~N} \mathrm{~m} \mathrm{rad}^{-1}$ and $k_{c}=300 \mathrm{~N} \mathrm{~m}^{-1}$. No thrust discretization or thrust distribution envelope are considered. A simplified fuel-consumption model has been considered for comparing the nominal differences between the control strategies. The consumed fuel $c=c_{\text {tras }}+c_{\text {rot }} \in \mathbb{R}$ is calculated as

$$
c_{\text {tras }}=\alpha \int_{0}^{t_{f}} \sum_{i=1}^{3}\left|f_{b, i}\right| \mathrm{d} t, \quad c_{\text {rot }}=\alpha \int_{0}^{t_{f}} \sum_{i=1}^{3}\left|\tau_{b, i}\right| \mathrm{d} t,
$$

where $c_{\text {tras }} \in \mathbb{R}$ and $c_{\text {rot }} \in \mathbb{R}$ are the amounts of fuel consumed by the thrusters for the translation and the rotation, respectively, and where $\alpha \in \mathbb{R}$ is a thruster-related coefficient. Here $\alpha=1 \mathrm{~s} \mathrm{~m}^{-1}$ is used for the sake of comparison.

Fig. 2 shows the end-effector position, the base position, the base attitude, the overall CoM position and the angular momentum for the three strategies. Fig. 3 shows the base force and base torque. Fig. 4 shows the fuel consumption.

In Fig. 2, the main functional difference on base translation and rotation among all strategies are recovered. The base position is kept constant for the full base control but changes and converges to new final values for the partial base and the floating-base controls. Meanwhile, the CoM position is kept constant for the partial base and the floating-base controls, but changes for the full base control. This indicates that the full base strategy displaces the system CoM during end-effector maneuvering even if no contact is involved, resulting in fuel inefficiency. On the other hand, for both partial base and floating-base controls the CoM is not displaced and this comes at no effort as the CoM automatically conserves due to the natural decoupling of the CoM dynamics. This is confirmed by the fact that for the partial base and floating-base strategies no base force is commanded, as observed in Fig. $3 \mathrm{~b}$ and Fig. $3 \mathrm{C}$ The base angles are kept constant for the full base and the partial base controls but change and converge to new values for the floating-base control. Conversely, the angular momentum is kept at zero for the floating-base control, but changes for the full base and the partial base controls. This indicates that the full base and partial base strategies vary the total angular momentum during end-effector maneuvering, and this results in fuel consumption. On the other hand, for the floating-base control, the angular momentum stays at zero and this comes at no effort as the angular momentum automatically conserves due to the natural decoupling of the angular momentum dynamics. This is confirmed by the fact that for floating-base
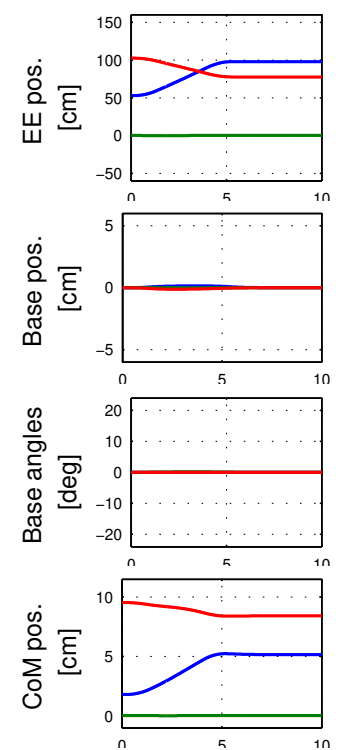

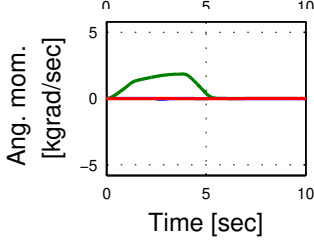

(a) Full base coordinated control
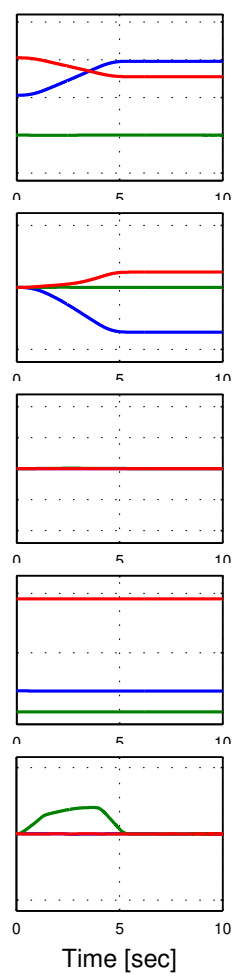

(b) Partial base coordinated control
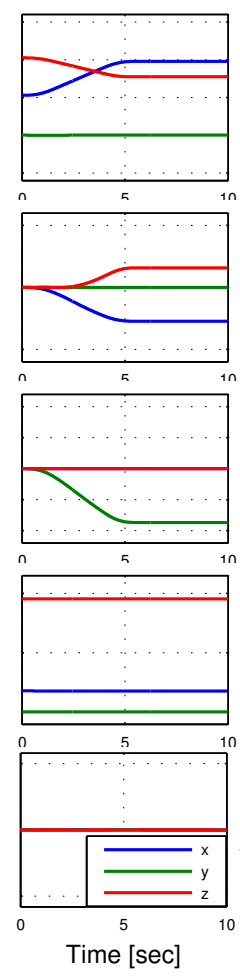

(c) Floating-base control
Fig. 2: Time-response for different control strategies.

control, no base torque is commanded, as shown in Fig. 3c The expected results regarding the fuel consumption are confirmed in Fig. 4a Therein, it is shown that the fuel consumption of the full base control is all the time bigger than that of the partial base and floating-base controls. Similarly, the fuel consumption of the partial base control is all the time bigger than that of the floating-base control, which is exactly zerd 3 . The total amount of consumed fuel is represented in further detail in Fig. 4b wherein the differences in rotation and translation are highlighted. In the figure it is possible to observe that the consumption can be greatly reduced when the requirements on the base control during end-effector operations are loosened. This improved fuel consumption is one advantage of the proposed partial base control compared to the full base control. Ideally, the consumption can be reduced to zero when both position and attitude requirements are loosened, as in the case of floating-base control. In scenarios in which the attitude control is mandatory, one may use the proposed partial base strategy instead of a full base control as an intermediate solution to save at least the considerable amount of fuel required for translation. The cost to pay is a displacement of the base position after end-effector maneuvers.

\section{EXPERIMENTAL VALIDATION}

The control method has been validated on the On-Orbit Servicing Simulator (OOS-Sim) hardware-in-the-loop facility [14]

\footnotetext{
${ }^{3}$ The small nonzero value in Fig. $4 \mathrm{~b}$ can be explained as drift of the simulation's discrete integrator.
} 


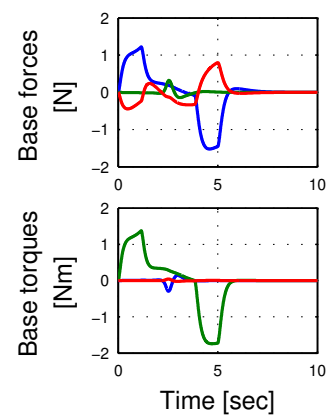

(a) Full base coordinated control
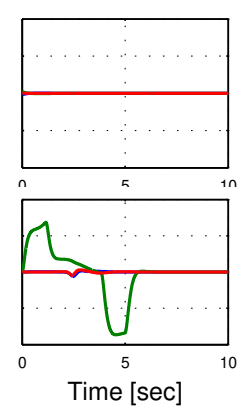

(b) Partial base coordinated control
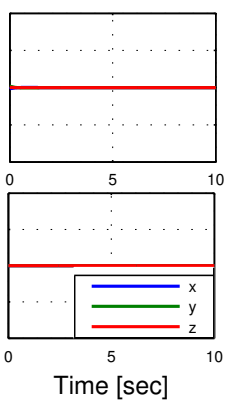

(c) Floating-base control
Fig. 3: Base commands for different control strategies.

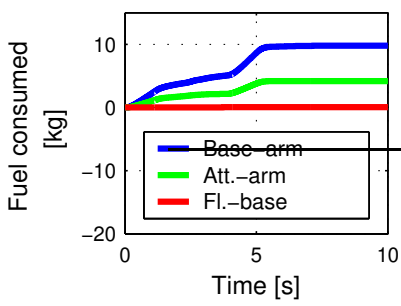

(a) Consumption vs. time.

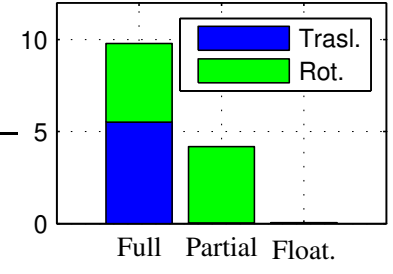

(b) Contribution of translation and rotation to total fuel.
Fig. 4: Fuel consumption for different control strategies.

at the DLR (see Fig. 5). The OOS-Sim is a robotic simulator for space robots, which enables the testing of space arms on ground before their actual deployment in orbit. The test arm is mounted on a simulator arm in a micro-macro configuration. The simulator arm reproduces the spacecraft's dynamics based on a real-time model integration. The test arm is a torquecontrolled KUKA KR4+ lightweight robot with seven degrees of freedom and the simulator arm is a position-controlled KUKA KR120 industrial robot. The microgravity conditions in the test arm are replicated by actively compensating the joint gravity torques based on an identified model. The space robot controller runs at a $1 \mathrm{~ms}$ rate. With this system, the space robot controller can be simulated taking into account real dynamics, sensor noise, time delay, control discretization, and model uncertainties of the test manipulator. Conversely,

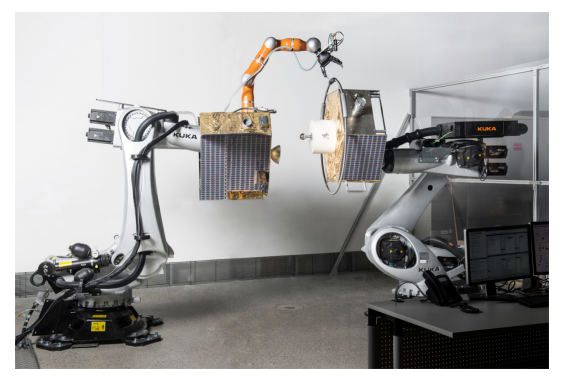

Fig. 5: The On-Orbit Servicing Simulator at DLR RMC. the spacecraft dynamics simulation is model-based. In the experiment, the same parameters and gains described in Sect $\mathrm{V}$ are used.

\section{A. Controller implementation}

The controller requires a model of the inertia of the space robot. The quantities $\boldsymbol{p}_{b c}$ and $\overline{\boldsymbol{J}}_{v}$ required in (32) can be extracted from the inertia matrix by using $(5 \mathrm{~b})$. The state in (28), (29), and (30) could be reconstructed as follows: $\tilde{\boldsymbol{x}}_{b}$, by using a spacecraft-mounted LIDAR; $\boldsymbol{\omega}_{b}$, by using a spacecraftmounted gyro; $\tilde{\boldsymbol{x}}_{e}$ and $\boldsymbol{\nu}_{e}$, by using the forward kinematics of the space robot or a camera mounted on the end-effector; $\boldsymbol{v}_{c}$, from (10), wherein $\boldsymbol{v}_{b}$ could be obtained by derivation of the LIDAR position or by fusion with other sensors for better performance. Finally, $\tilde{\boldsymbol{x}}_{c}$ could be obtained from the LIDAR position. In the present test, no LIDAR, gyro or cameras are used and the states are simplistically reconstructed from the forward kinematics of the test and simulation arms. Noise on $\boldsymbol{v}_{c}$ in (10) affects $\boldsymbol{f}_{c}$ in (28) and in turn $\boldsymbol{f}_{b}$ in (32), causing unnecessary thrust activation. To cope with that, in the present work a deadzone of $2 \mathrm{~N}$ is used on the $f_{b}$ signal. As a final remark, the proposed controller is subject to the singularities of the Jacobian $\boldsymbol{J}_{e}^{\oplus}$. At singularity the algorithm does not fail computationally but only results in loss of actuation in a singular direction.

\section{B. Experimental results}

A sequence of two representative end-effector maneuvers in a grasping scenario was tested. For each maneuver, the endeffector is commanded to reach a desired pose and then to return to the initial position. In the second maneuver, a lateral motion (y component) of the end-effector is commanded to excite three-dimensional effects more pronouncedly. In both maneuvers, the base attitude and the CoM were commanded to hold desired setpoints. No contact or initial momentum were simulated. In order to validate the statements regarding the decoupling properties of the proposed approach, the experiment was performed first with the proposed decoupled actuation (32). Then, the experiment was repeated with a control that enforces exactly the same requirements but with the coupled actuation (33).

Fig. 6) shows the time responses of the end-effector position, the base attitude, and the CoM position. The end-effector successfully converged to the desired position in both cases. The base attitude was slightly displaced due to the robot motion, but the control action successfully restored it after the maneuver ended. With the decoupled actuation, the CoM stayed in place 4 and was not affected by the end-effector control. Conversely, with coupled actuation the CoM was excited by the coupling term of the end-effector control input $\boldsymbol{w}_{e}$ in (33) into the base actuator $f_{b}$. Interestingly, for decoupled actuation the base position returned to the initial position after the maneuvers ended. Fig. 7 shows the commanded base force and base torque. Therein, the main result of the proposed

${ }^{4}$ Small deviations from zero that can be explained as disturbances induced by the hardware simulation facility. 


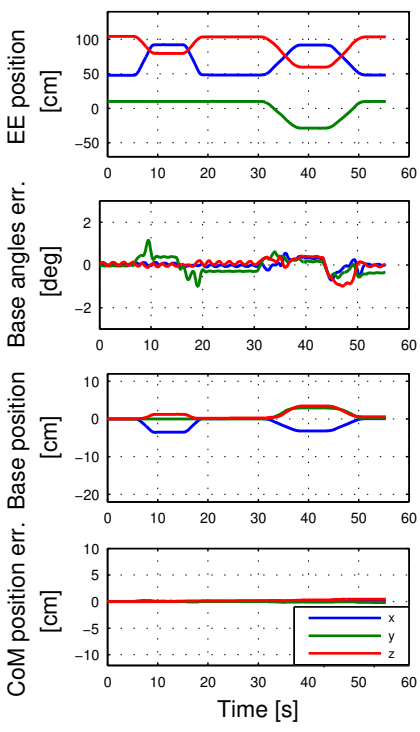

(a) Decoupled actuators (proposed)

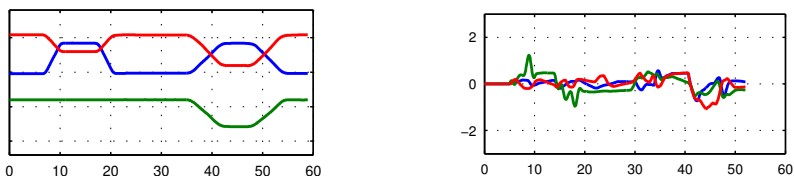

(a) Base angles $[\mathrm{deg}]$

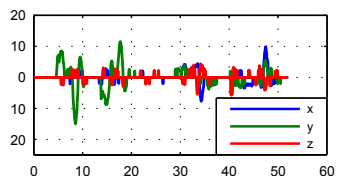

(b) Base torques $[\mathrm{Nm}]$

Fig. 8: Test with deadzone on $\tau_{b}$ (decoupled actuator).

\section{CONCLUSIONS AND FUTURE WORKS}

The problem of the simultaneous control of the base attitude and the end-effector pose of a space robot has been addressed. A dynamics decomposition has been proposed that decouples the end-effector task from the base force actuator and reduces the thrusters use. A simulation study has been conducted to highlight the fuel-efficiency advantage of the proposed partial control compared to full-base control. Hardware experiments successfully validated the method. Future works may validate it with real thrusters and sensors models and with a larger set of grasping tasks, and may investigate the performance increase due to reduced saturation.

\section{REFERENCES}

[1] Z. Vafa and S. Dubowsky, "The kinematics and dynamics of space manipulators: The virtual manipulator approach," The International Journal of Robotics Research, vol. 9, no. 4, pp. 3-21, 1990.

[2] Y. Umetani and K. Yoshida, "Continuous path control of space manipulators mounted on omv," Acta Astronautica, vol. 15, no. 12, pp. 981 986, 1987.

[3] Y. Masutani, F. Miyazaki, and S. Arimoto, "Sensory feedback control for space manipulators," in Robotics and Automation, IEEE International Conference on, May 1989, pp. 1346-1351 vol.3.

[4] A. M. Giordano, G. Garofalo, and A. Albu-Schaffer, "Momentum dumping for space robots," in 2017 IEEE 56th Annual Conference on Decision and Control (CDC), Dec 2017, pp. 5243-5248.

[5] A. M. Giordano, D. Calzolari, and A. Albu-Schaffer, "Workspace fixation for free-floating space robot operations," in 2018 IEEE International Conference on Robotics and Automation (ICRA), May 2018.

[6] D. Nenchev, K. Yoshida, and Y. Umetani, "Analysis, design and control of free-flying space robots using fixed-attitude-restricted jacobian matrix," pp. 251-258, 011991.

decomposition is evident, namely, the base force was exactly zero for decoupled actuation, in agreement with the observed zero CoM error. In contrast, the base force was nonzero for coupled actuation. This zero base force is the main property that leads to improved fuel consumption. The experiments were repeated three times:

1) The same trajectories were tested one more time for repeatability. The same results were obtained and are not shown for brevity.

2) The same path with a longer duration was commanded. Similar results were obtained and are not shown.

3) The same trajectory was repeated with an additional deadzone of $2 \mathrm{Nm}$ on the $\tau_{b}$ signal. In Fig 8 the base angles and commanded base torques are reported. Therein it is shown that the deadzone avoided the steady oscillation on the base torque observed in Fig 7 without inducing stability issues. Other plots showed no significant difference and are not reported.

In conclusion, the experimental results validate the effectiveness of the proposed control and prove the fuel-efficiency advantages as a consequence of the decoupled actuation.

[7] Y. Xu, H. Y. Shum, J. J. Lee, and T. Kanade, "Adaptive control of space robot system with an attitude controlled base," in Proceedings 1992 IEEE International Conference on Robotics and Automation, May 1992, pp. 2005-2010 vol.3.

[8] F. Aghili, "Coordination control of a free-flying manipulator and its base attitude to capture and detumble a noncooperative satellite," in 2009 IEEE/RSJ International Conference on Intelligent Robots and Systems, Oct 2009, pp. 2365-2372.

[9] E. Papadopoulos and S. Dubowsky, "Coordinated manipulator/spacecraft motion control for space robotic systems," in Proceedings. 1991 IEEE International Conference on Robotics and Automation, Apr 1991, pp. 1696-1701 vol.2.

[10] R. M. Murray, Z. Li, and S. S. Sastry, A Mathematical Introduction to Robotic Manipulation, 1st ed. Boca Raton, FL, USA: CRC Press, Inc., 1994.

[11] C. Ott, Cartesian impedance control of redundant and flexible-joint robots. Springer, 2008.

[12] C. Natale, Interaction control of robot manipulators: six degrees-offreedom tasks. Heidelberg: Springer, 2003, vol. 3.

[13] M. I. El-Hawwary and M. Maggiore, "Reduction theorems for stability of closed sets with application to backstepping control design," Automatica, vol. 49, no. 1, pp. $214-222,2013$.

[14] J. Artigas, M. D. Stefano, W. Rackl, R. Lampariello, B. Brunner, W. Bertleff, R. Burger, O. Porges, A. Giordano, C. Borst, and A. AlbuSchaeffer, "The OOS-SIM: An on-ground simulation facility for on-orbit servicing robotic operations," in 2015 IEEE International Conference on Robotics and Automation (ICRA), May 2015. 